\title{
Review \\ A Potential Benefit of Hypochlorous Acid - Facial Sanitisation: A Review
}

\author{
Avis Aman Nowbuth ${ }^{1, t, \ddagger} \mathbb{\infty}$, Josh Barrie Armstrong ${ }^{1, \ddagger}$, Thomas Eugene Cloete ${ }^{2, *}$ and Pieter Fourie ${ }^{2, *}$ \\ 1 BSafe HOCl; nowbuthaman@gmail.com; josharmstrong149@gmail.com \\ 2 Department of Microbiology, Stellenbosch University; eugenecloete@sun.ac.za \\ 3 Innovation4Life; pieter@innovation4life.com \\ * Correspondence: nowbuthaman@gmail.com (A.A.N.); josharmstrong149@gmail.com (J.B.A.); \\ eugenecloete@sun.ac.za (T.E.C.); pieter@innovation4life.com (P.F.) \\ $\ddagger$ These authors contributed equally to this work.
}

\begin{abstract}
Sanitisation has become a major component of everyday life, with emphasis on the hands and surfaces. The face remains unsanitised. This is due to the lack of an acceptable sanitiser. The use of masks has been implemented in order to protect the spread of the pathogens by covering the face, however there remain issues associated with the use of PPE.. The face remains a harbour for upper respiratory tract infections, with constant deposition and replication of microbes. With SARS-CoV-2 being so prominent, it was postulated that there is an inoculum dose-dependant relationship with severity. By reducing microbial load of the face, the risk of both infection and severity of infection are reduced. $\mathrm{HOCl}$ has proven antimicrobial and antiinflammatory activity and was tested for efficacy against SARS-CoV-2, demonstrating a 99.99\% reduction (50ppm; 1 min contact). A facial sanitiser, added to the arsenal of hand sanitisers and masks, further improves protection and prevention against SARS-CoV-2. The advantages of regular sanitising of the face and mask include a reduced level of microbial contamination, reduced risk of biofilm formation, and respiratory tract and skin infections. $\mathrm{HOCl}$ was reviewed as a face and face mask sanitiser, concluding that it was an ideally suited product.
\end{abstract}

Keywords: $\mathrm{HOCl}$; hypochlorous; antimicrobial; antiinflammatory; SARS-CoV-2; infections; sanitisation

\section{Introduction}

The face, namely through the oral and nasal mucosa and the conjunctiva, is a major point of entry and point of infection for many pathogens involved in human diseases and serves as a harbour for the growth of microorganisms. This is particularly true for SARS-CoV-2, among other significant infections (e.g., S. pyogenes, S. aureus, M. tuberculosis) involving the respiratory tract. The pathogenic contamination of the face is by both airborne pathogens and surface borne pathogens, and via unsanitised hands. On average, individuals who are well-educated on infection control involuntarily touch their faces 23 times per hour where $44 \%$ of the face touches involved mucosal areas, with the remainder $56 \%$ being non-mucosal surfaces. [1]

With the ongoing Covid-19 pandemic, regular hand and surface sanitisation and regular hand washing has been emphasised in order to reduce cross contamination and risk of infection. While this is beneficial, involuntary face touching, especially around the mucosal regions, alongside the presence of airborne pathogens, increases the risk of infection. Consequently, the use of disposable or reusable face masks has been made mandatory. Although it is true that the use of a face mask is beneficial in reduction of the risk of infection, there remains certain issues with use of the face mask - the main issues being improper usage of face masks, prolonged usage of disposable face masks, and infrequent and inadequate washing of reusable masks. It should also be considered that poor handling of masks and frequent removal and reapplication of the same masks further increases the surface area of the face mask which is contaminated.

As face masks only cover the nose and mouth, there remains an exposure risk of the eyes 
and conjunctiva. This poses a risk of contact and entry for pathogens even whilst wearing a mask. In addition to this, upon removal of face masks and other personal protective equipment (PPE), there is still the risk of contamination of skin surfaces. Errors in doffing (removal) procedures of PPE was studied by Okamato et. al. (2019) whereby 125 participant healthcare workers (HCW) were enrolled. The findings demonstrated that $39.2 \%$ made multiple doffing errors and $36 \%$ were found to be contaminated with multidrug resistant organisms. [2] Lim et. al. (2015) performed a study in Samsung Medical Center in South Korea, whereby they identified a $20.6 \%$ error rate of donning of respirators or hoods. Contamination on the doffing process identified a $48.3 \%$ rate of contamination of the head, $6.9 \%$ contamination rate of the face, and $72.4 \%$ rate of contamination of the neck. [3]

Even though it is clear that the primary focal point of many infections is the face and facial mucosal surfaces, there is a need for disinfection products that can be used to sanitize the face and to rid the face and mucosal surfaces of pathogens. A facial sanitiser would be a great tool to add to the current arsenal against the Covid-19 pandemic, as well as against other respiratory tract infections. $\mathrm{HOCl}$ is a secondary reactive oxygen species produced by neutrophils, macrophages, and masts cells. Physiologically $\mathrm{HOCl}$ can be said to be present in all tissue systems, including the skin. [4]

$\mathrm{HOCl}$ has proven to be a highly effective disinfectant, demonstrating rapid activity against a wide spectrum of microbes, including drug resistant bacteria and bacterial spores, fungi, viruses, prions, and amyloid seeds. [5,6]

In this review, the mechanism of action of $\mathrm{HOCl}$ will be briefly discussed alongside the safety of use of $\mathrm{HOCl}$ on sensitive areas such as the face. The potential benefits of using $\mathrm{HOCl}$ to reduce the number of pathogens will be discussed, and other potential benefits which may be associated with the use of $\mathrm{HOCl}$ will be identified.

\section{The Antimicrobial Mechanism of Action and Safety of $\mathrm{HOCl}$}

\subsection{Mechanism of Action}

Hypochlorous acid is a secondary reactive oxygen species (ROS). It is a naturally occurring molecule present in the human body, produced during the respiratory burst of phagocytosis. The composition of the molecule is $\mathrm{HOCl}$ - the molecule itself being the secondary reactive oxygen species. The molecule then dissociates into free radicals $-\mathrm{OH}-$ and $\mathrm{Cl}+\mathrm{OH}$ - is a potent free radical (oxidant) which rapidly causes damage to bacterial cell walls and cell contents. The following summarises the antimicrobial action of $\mathrm{HOCl}$ : [7]

1. Hydroxyl ions (OH-) react with membrane lipids and cell walls of pathogens resulting in:

- $\quad$ Peroxidation of polyunsaturated fatty acids and destruction of cross-linkages in cell walls

- A chain reaction of peroxidation of membrane lipids and decreased membrane fluidity

2. Cellular dysfunction occurs due to inhibition of enzymes required for glycolysis, thus, pathogens, specifically bacteria, are unable to metabolise glucose to produce ATP resulting in:

- An increase in the presence of free radicals, resulting in a change in intracellular redox potential further inhibiting glycolysis

- The complete disruption of essential cellular functions and depletion of adenosine triphosphate (ATP)

3. Additionally, there is activation of enzymes resulting in cellular lysis

Human cells make use of a group of defence mechanisms collectively known as the Antioxidant Defence System (ADS). [8] Microbial cells, however, do not have this defence mechanism and for this reason $\mathrm{HOCl}$ is effective against microbes. 


\subsection{Safety}

The potential for $\mathrm{HOCl}$ to damage mammalian cells appears to be present. However, inherent mechanisms in human cells are in place in order to prevent cellular damage. The physiological protective mechanism is based on the antioxidant defence mechanism. As $\mathrm{HOCl}$ is a ROS (oxidant), the antioxidant defence mechanism of human cells protects against the oxidative stress caused by $\mathrm{HOCl}$. This specific quality is what renders $\mathrm{HOCl}$ safe for use in humans while making it toxic to microbes. SkinSafe, developed by Mayo Clinic, designates $\mathrm{HOCl}$ as hypoallergenic, irritant free, eyelid and lip safe, safe for teens and safe for babies. [9]

Antioxidants are molecules which slow down or prevent oxidation of other molecules. The mechanism of action of antioxidants are as follows [10]:

1. Removal of radical intermediate species

2. Blocking secondary production of toxic metabolites and inflammatory mediators

3. Converting free radicals into less toxic compounds

4. Blocking chain propagation of secondary radicals

5. Repairing molecular injury

6. Enhancing the endogenous antioxidant system function of exogenous antioxidants

7. Inhibiting other oxidation reactions by being oxidised themselves

As such, there are two classes of antioxidants - enzymatic and non-enzymatic antioxidants. Enzymatic antioxidants include superoxide dismutases, catalases, glutathione system, thioredoxin system. Non-enzymatic antioxidants include ascorbic acid (vitamin C), glutathione and thiocyanate, tocopherols and tocotrienols (Vitamin E), beta-carotene (Carotenoids - provitamin A).

The Antioxidant Defence System (ADS) is what protects the body from oxidative damage. The presence of the ADS alone makes $\mathrm{HOCl}$ safe for use in humans and other species that make use of this kind of system.

\subsection{The Skin Microbiome}

The skin is the human body's largest organ and is colonised by a plethora of microorganisms. This colonisation is highly dependent on topographical location, host, and environmental factors. [11] The skin is the body's primary defence mechanism against microbial infection, acting as a physical barrier. The skin on the face is particularly susceptible to colonisation by foreign microbes, due to frequent face touching and deposits of airborne microbes (droplets) on the skin and mucosa of the face. Many common skin disorders are postulated (although having not been scientifically proven in most cases) to have an underlying microbial cause because clinical improvement is seen with the use of antimicrobial treatments. However, there are certain skin disorders, such as acne vulgaris, with definitive causative microorganisms (e.g., Cutibacterium acnes (formally Propionibacterium acnes) and Staphylococcus epidermidis). [12] The presence of Staphylococcus aureus on the skin and scalp in itself is a cause of many infections, such as boils, folliculitis, impetigo, and cellulitis. Additionally, S. aureus is highly prevalent in cases of atopic dermatitis (eczema). [13] S. aureus, in addition with group A streptococcus (S. pyogenes), and other microorganisms, can cause a severe polymicrobial infection - necrotising fasciitis. As such, microorganisms on the face may pose a threat of a range of skin infections ranging from minor easy to treat infections to severe life-threatening infections. [14]

The variety of bacteria which form the microbiome of the face may create biofilms. This begins with microorganisms forming a microcolony and surface colonisation. Biofilms provide advantages to microorganisms including: protection from host defences, differential gene expression, and increased resistance to antimicrobials Epithelial biofilms are implicated in a number of dermatological conditions, such as chronic wounds, atopic dermatitis, candidiasis, and acne vulgaris. These biofilms pose a challenge, as there are limitations for their detection, and their resistance to conventional antimicrobial therapy due to persistence and their chronic nature. $[15,16]$ 


\subsection{The Face as a Harbour for Respiratory Infections}

The entry points to the nose, mouth and eyes are key regions which predispose infections of the upper respiratory tract. Microorganisms proliferate on the surface of the skin, and constant touching of the face, nose, mouth, and eyes results in an increase in presence of the number and variety of microorganisms present. The major causative agents for upper respiratory tract infections (URTIs) are viral in nature, although not limited to viruses alone. [17]

Microorganims involved in the pathogenesis of upper respiratory tract infections include rhinovirus, respiratory syncytial virus (RSV), parainflenza virus, coronavirus, influenza viruses, adenovirus, enterovirus, S. aureus, S. pneumoniae, H. influenzae, P. aeruginosa, M. catarrhalis, E. coli, K. pneumoniae, N. gonorrhoeae, among other pathogens.

SARS-CoV-2 is a major pathogen of interest in recent times which causes upper respiratory tract infections. Wim Van Damme et. al.(2021) discuss the relationship of inoculum dose and disease severity of SARS-CoV-2 due to three possible factors:

1. At an individual level: "viral dose in inoculum is related to severity of disease (dosedependent relationship)"

2. At a cluster level: "Severity of disease is related to transmission potential" leading to clusters of mild cases and clusters of severe cases

3. At a community level: "In certain contexts, chains of severe cases can build up through intensive transmission with high inoculum to severe local outbreaks, which can result in large-scale intensive epidemics, while this is less likely in other contexts"

This theory plays out in practice on three levels: [18]

1. 1. Individual level: A person infected with a small dose viral inoculum will on average develop milder disease than a person infected with a high viral inoculum and vice versa. This is independent of other well known risk factors for severity of disease, mainly old age, and comorbidities, such as diabetes.

2. Cluster level: A person with asymptomatic infection or mild disease, will on average spread lower dose of the virus, and is less likely to transmit disease; and when the person transmits, the newly infected person is more likely to have a mild disease compared to a person infected by a severely ill person, who spreads on average higher doses of the virus. This causes clusters and chains of milder cases or more severe cases.

3. Community level: In certain contexts, such as dense urban centres with a moderate climate, during the season that people live mostly indoors, the potential for intensive transmission and explosive outbreaks is higher than in rural areas, or in regions with a hot and humid climate where people live mostly outdoors. Hence, a cascade of intensive transmission is more likely in certain contexts than others.

This model is based on other pathogens which show dose-dependent severity, such as:

- Influenza virus

- Coronavirus (seen with MERS and HCoV-229E)

- Human immunodeficiency virus (HIV)

- Measles

- Mycobacterium tuberculosis (TB)

- Streptococcus pneumoniae

It is of note that this hypothesis needs further investigation to in order to be well established, and medical experts need to pay close attention to these factors.[18]

\section{Dermatological Benefits of Hypochlorous Acid}

As the use of alcohol-based sanitisers has increased drastically since the onset of the Covid-19 pandemic, resulting in an increase in the incidence of contact dermatitis and allergic contact dermatitis associated with the use of alcohol-based sanitisers.[19] The incidence 
of allergic contact dermatitis increased with use of other non-alcohol-based hand sanitisers (such as chlorhexidine and quaternary ammonium compounds). Another concern with the use of alcohol-based sanitisers is the depletion of the lipid barrier with repeated exposure, resulting in increased risk of destruction or changes of the skin flora and colonisation of pathogens.[20]

Additionally, the use of personal protective equipment (PPE) has shown an increase in dermatological conditions such as allergic dermatitis, irritant dermatitis, friction blisters, contact urticaria, acne, and infections.[21]

$\mathrm{HOCl}$ is a possible solution to the aforementioned conditions, while possessing other benefits associated with dermatological applications. In a mouse model, anti-inflammatory properties were demonstrated on sensitised mice, whereby $\mathrm{HOCl}$ demonstrated effective reduction in an inflammatory response quantified by reduced secretion of inflammatory cytokines. Associated with this was a reduction in itch and scratching behaviour. [22]

The antimicrobial and anti-inflammatory (immunomodulatory) properties of $\mathrm{HOCl}$ correlate with a clinical improvement in a variety of cutaneous disorders - including atopic dermatitis, seborrheic dermatitis, diabetic ulcers, pruritis, and acne vulgaris. Other clinical benefits are demonstrated with the promotion of wound healing and reduction or prevention of scar formation. [23]

\section{Potential Benefits of $\mathrm{HOCl}$ on the Face and Skin}

Research in the field of facial sanitisation is extremely inadequate even though the potential benefits are innumerable. Due to the high efficacy of $\mathrm{HOCl}$ and the inherent safety profile, it offers very good potential for use for face as well as nasal and oral sanitisation. $\mathrm{HOCl}$ acts as secondary reactive oxygen species when produced endogenously and this is the way it carries out its mechanism of action. Due to its own endogenous nature, $\mathrm{HOCl}$ possesses an inherently good safety profile as demonstrated in numerous international studies.[24-27]

Testing of antimicrobial efficacy of $\mathrm{HOCl}$ has been sponsored by Aquaox LLC, Loxahatchee, Florida, on multiple pathogens following the USP $<51>$ testing protocol.[28]

Relevant pathogens tested include Candida albicans, S. aureus, and S. epidermidis demonstrated a reduction of $>99.9999 \%$ with 15 seconds of contact. P. aeruginosa showed a reduction of $>99.9999 \%$ with 60 seconds of contact and MRSA had a reduction of $>99.999 \%$ with 60 seconds of contact. The 2009 Pandemic strain of H1N1 had a $>99.963 \%$ reduction with 5 minutes of contact, and M. bovis demonstrated a $>99.99 \%$ reduction with 10 minutes of contact.

From the above results it is clear that Hypochlorous Acid $(\mathrm{HOCl})$ will offer an additional defence mechanism to prevent microbial infection.

\section{Discussion and Conclusion}

The published literature indicates that $\mathrm{HOCl}$ is highly efficacious against a wide range of microorganisms. This, alongside knowledge of the mechanism of action provides insight into the antimicrobial properties which may bypass the potential of microbial resistance against $\mathrm{HOCl}$. The range of microorganisms killed by $\mathrm{HOCl}$ are all significant microbes which cause dermatological or respiratory tract infections. As such, it can be deduced that use of $\mathrm{HOCl}$ as a facial and face mask sanitiser would decrease incidence of the skin conditions mentioned. The safety of $\mathrm{HOCl}$ to mammalian cells has been demonstrated even the most sensitive cells. As $\mathrm{HOCl}$ with a concentration of $200 \mathrm{ppm}(0.2 \%)$ and $210 \mathrm{ppm}$ $(0.21 \%)$ has been proven safe using Vero cells, it can be concluded that $\mathrm{HOCl}$ is a safe to use as a facial sanitiser.[27] In addition to the benefits of preventing upper respiratory tract infections, $\mathrm{HOCl}$ has further benefits with the potential to treat a range of dermatological disorders, such as acne vulgaris, seborrheic dermatitis, and atopic dermatitis. Furthermore, a range of cutaneous conditions associated with the use of PPE and alcohol-based sanitisers can see a benefit from the use of $\mathrm{HOCl}$. As such, the combined benefits of $\mathrm{HOCl}$ use further potentiates the use of the compound as a facial sanitiser. $\mathrm{HOCl}$ has a proven efficacy against 
biofilms, and as such, the presence of epithelial biofilms would be eradicated, alongside conditions associated with them. Use of a facial sanitiser, in addition to the arsenal of hand sanitiser and masks, further improves protection against and prevention of the spread of SARS-CoV-2. The inoculum dose-dependent relationship with severity of infection postulation brings to consideration that any reduction of SARS-CoV-2 numbers on the face and mucosal surfaces will be beneficial with reduction of both risk of infection and severity of ongoing infection.

Author Contributions: Conceptualization, A.A.N. and J.B.A.; Writing—original draft preparation, A.A.N, J.B.A.; writing-review and editing T.E.C.,P.F.

Funding: Funding was provided by BSafeHOCl

Conflicts of Interest: Dr. Nowbuth and Dr. Armstrong are employees of BSafe HOCl. The funders had no role in the review.

$\begin{array}{ll}\text { Abbreviations } \\ \text { The following abbreviations are used in this manuscript } \\ \text { MDPI } & \text { Multidisciplinary Digital Publishing Institute } \\ \text { DOAJ } & \text { Directory of open access journals } \\ \text { TLA } & \text { Three letter acronym } \\ \text { LD } & \text { Linear dichroism } \\ \text { HOCl } & \text { Hypochlorous acid } \\ \text { PPE } & \text { Personal protective equipment } \\ \text { HCW } & \text { Healthcare workers } \\ \text { OH- } & \text { Hydroxyl ions } \\ \text { ATP } & \text { adenosine triphosphate } \\ \text { ADS } & \text { Antioxident defence system } \\ \text { ROS } & \text { Reactive oxidant species } \\ \text { URTI } & \text { Upper respiratory tract infection } \\ \text { MERS } & \text { Middle East respiratory syndrome }\end{array}$

\section{References}

1. Kwok, Yen Lee Angela; Gralton, Jan; McLaws, Mary-Louise, Face touching: a frequent habit that has implications for hand hygiene Am. J. Infect. Control 2015, 112-114.

2. Okamoto, Koh; Rhee, Yoona; Schoeny, Michael; Lolans, Karen; Cheng, Jennifer; Reddy, Shivani; Weinstein, Robert A.; Hayden, Mary K.; Popovich, Kyle J., Impact of doffing errors on healthcare worker self-contamination when caring for patients on contact precautions Infect. Control Hosp. Epidemiol. 2019, 559-565.

3. Lim, Seong Mi; Cha, Won Chul; Chae, Minjung Kathy; Jo, Ik Joon, Contamination during doffing of personal protective equipment by healthcare providers Clin Exp Emerg Med 2015, 162-167.

4. Degrossoli, Adriana; Muller, Alexandra; Xie, Kaibo; Schneider, Jannis F; Bader, Verian; Winklhofer, Konstanze F; Meyer, Andreas J; Leichert, Lars I, Neutrophil-generated HOCl leads to non-specific thiol oxidation in phagocytized bacteria eLife 2018

5. Eryılmaz, Mujde; Palabiyık, Ismail Murat, Hypochlorous Acid - Analytical Methods and Antimicrobial Activity Trop. J. Pharm. Res. 2016, 123-126

6. Hughson, Andrew G; Race, Brent; Kraus, Allison; Sangare, Laura R.; Robins, Lori; Groveman, Bradley R.; Saijo, Eric; Phillips, Katie; Contreras, Luis; Dhaliwal, Virkamal; Manca, Matteo; Zanusso, Gianluigi; Terry, Daniel; Williams, Jeffrey F.; Caughey, Byron, Inactivation of Prions and Amyloid Seeds with Hypochlorous Acid PLoS Pathogens 2016

7. Dobyns, E. L.; Stenmark, K. R. Kendig's Disorders of the Respiratory Tract in Children, 7th ed.; Elsevier: Philidelphia, USA, 2006; pp. 224-242.

8. Birben, Esra; Sahiner, Umit Murat; Sackesen, Cansin; Erzurum, Serpil; Kalayci, Omer, Oxidative Stress and Antioxidant Defense WAOjournal 2012, 9-19

9. SkinSafe. Available online: https://www.skinsafeproducts.com/ingredients/hypochlorous-acid (accessed on 10 May 2021)

10. Kabel, Ahmed M., Free Radicals and Antioxidants: Role of Enzymes and Nutrition J. Nutr. Health 2014, 35-38

11. Grice, E. A.; Segre, J. A., THe Skin Microbiome Nat. Rev. 2011, 244-253

12. Bek-Thomsen, M.; Lomholt, H. B.; Kilian, M., Acne is Not Associated with Yet-Uncultured Bacteria J. Clin. Microbiol. 2008, 3355-3360

13. Tong, Steven Y. C.; Davis, Joshua S.; Eichenberger, Emily; Holland, Thomas L.; Fowler, Vance Jr. G., Staphylococcus aureus Infections: Epidemiology, Pathophysiology, Clinical Manifestations, and Management Clin. Microbiol. Rev. 2015, 603-661 
14. Yamamato; Loren; MD; MPH, Treatment of Skin and Soft Tissue Injuries Pediatr. Emerg. Care 2017, 49-55

15. Vaishnavi, Kambiam Veettil; Safar, Lulua; Devi, Keerankulangara, Biofilm in Dermatology J. Skin Sex. Transmitted Dis. 2019

16. Brandwein, Michael; Steinberg, Doron; Meshner, Shiri, Microbial biofilms and the human skin microbiome npj 2016

17. Dasaraju, P V; Liu, C Medical Microbiology, 4th ed.; University of Texas Medical Branch at Galveston: Galveston, USA, 1996.

18. Van Damme, Wim; Dahake, Ritwik; Van de Pas, Remco; Vanham, Guido; Assefa, Yibeltal, COVID-19: Does the infectious inoculum dose-response relationship contribute to understanding heterogeneity in disease severity and transmission dynamics? Med. Hypotheses 2021

19. Alves, S; Arendse, A; Kannenberg, S, COVID-19 collateral damage: Alcohol rub dermatitis as an emerging problem S. Afr. Med. J. 2020, 1148

20. Jing, Jane Lee Jia; Yi, Thong Pei; Bose, Rajendran J. C.; McCarthy, Jason R; Tharmalingam, Nagendran; Madheswaran, Thiagarajan, Hand Sanitizers: A Review on Formulation Aspects, Adverse Effects, and Regulations Int. J. Environ. Res. Public Health 2020, 3326

21. Sanghvi, Akanksha R., COVID-19: An overview for dermatologists Int. J. Dermatol. 2020, 1437-1449

22. Fukuyama, T; Martel, B. C.; Linder, L. E.; Ehling, S.; Ganchingco, J. R.; Baumer, W., Hypochlorous acid is antipruritic and anti-inflammatory in a mouse model of atopic dermatitis Clin. Exp. Allergy 2018, 78-88

23. Del Rosso, James Q; Bhatia, Neal, Status Report on Topical Hypochlorous Acid: Clinical Relevance of Specific Formulations, Potential Modes of Action, and Study Outcomes J Clin Aesthet Dermatol 2018, 36-39

24. Kubota, Akio; Goda, Taro; Tsuru, Tomomistu; Yonekura, Takeo; Yagi, Makoto; Kawahara, Hisayoshi; Yoneda, Akihiro; Tazuke, Yuko; Tani, Gakuto; Tomohiro, Ishii; Umeda, Satoshi; Hirano, Katsuhisa, Efficacy and safety of strong acid electrolyzed water for peritoneal lavage to prevent surgical site infection in patients with perforated appendicitis Surg. Today 2015, 867-879

25. Burian, Ewa A.; Sabah, Lubna; Kirketerp-Møller, Klaus; Ibstedt, Elin; Fazli, Magnus M.; Gundersen, Glenn, The Safety and Antimicrobial Properties of Stabilized Hypochlorous Acid in Acetic Acid Buffer for the Treatment of Acute Woulds - A Human Pilot Study and In Vitro Data Int. J. Low. Extrem. Wounds 2021, 1-9

26. Dissemond, Joachim, Wound cleansing: benefits of hypochlorous acid J. Wound Care 2020, S4-S8

27. Kabamba, Alexandre; Malatji, Kanyane Bridgett, Measuring the Inhibitory Activity of BSafe HOCl Samples 3 and 5 Against SARS-CoV-2 using in vitro Cell Based Assay, (CSIR Biosciences, Pretoria, South Africa), Report, 2020

28. Aquaox LLC, HOCl GLP Efficacy Data, (Aquaox LLC, Loxahatchee, Florida, USA), Report 